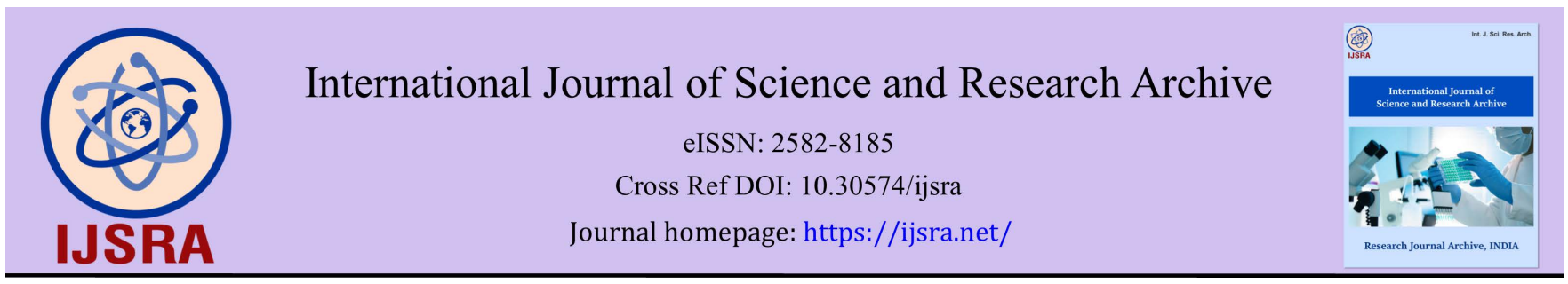

(CASE REPORT)

\title{
Reconstruction of a soft tissue defect of the thumb using a modified dorsal metacarpal artery flap: A case report study
}

\author{
Christos Konstantinidis, Christos Kotsias, Dimitrios Giotis *, Vasileios Panagiotopoulos, Konstantinos \\ Konidaris, Dimitrios Vardakas and Sotiris Plakoutsis
}

Orthopaedic Department, General Hospital of Ioannina “G. Hatzikosta”, Ioannina, Greece.

International Journal of Science and Research Archive, 2021, 03(02), 107-112

Publication history: Received on 10 August; revised on 23 September 2021; accepted on 25 September 2021

Article DOI: https://doi.org/10.30574/ijsra.2021.3.2.0147

\begin{abstract}
Finger injuries to the hand with extensive soft tissue defects might be challenging to treat. Typically, the first dorsal metacarpal artery (DMA) flap is used for reconstruction, whereas closure of the donor site is routinely performed with a split-thickness skin graft. The aim of the current study is to present a surgical technique that reports a modified incision, which allows elevation of the first DMA flap for the management of the soft tissue defects of the thumb without requiring a skin graft for coverage of the donor site. A 65-year-old man presented to the emergency department with a crush injury to the thumb. The patient had an extensive skin defect on the dorsal side of the thumb extending circumferentially to the ipsilateral thenar without tendon damages, whereas sensation of the finger was found intact. Surgical intervention was performed within six hours and involved DMA flap transfer through a modified skin incision, which allowed coverage of the donor defect in the index finger with a second DMA perforator flap. 3 months postoperatively, patient's satisfaction, sensation and range of motion (ROM) of the fingers were assessed using The Michigan Hand Outcomes Questionnaire (MHQ) and the 2-point discrimination (2-PD) test. The patient regained active $\mathrm{ROM}$ of the thumb and index along with complete preservation of sensation, while the final cosmetic outcome was satisfactory. The application of this technique allows coverage of both trauma defect and donor site with a single skin incision, providing a successful clinical outcome.
\end{abstract}

Keywords: Skin defect; Modified dorsal metacarpal artery flap; Thumb; Reconstruction; Skin graft.

\section{Introduction}

The multifunctional role of the thumb in nearly all human hand activities render it as the most significant finger [1]. Therefore, thumb injuries have much more important effect on hand function than other digit injuries [2]. Especially in cases which involve complex soft tissue defects of the thumb with exposure of bones and tendons, the reconstruction might be challenging due to limitations in local soft tissue availability and need for sensate skin coverage to restore thumb utility. Patients with permanent partial or fully thumb disability and dysfunction will have severe deficits in quality of life [2].

Required factors for a successful functional thumb reconstruction include the maintenance of adequate length, motility, stability and sensation [2]. Several reconstructive options such as local, regional and free flap transfers have been used including the dorsal metacarpal artery (DMA) flaps [2-4]. In particular, the use of the first DMA flap has been widely reported in the indexed literature for the repair of deep soft tissue defects of the thumb [5-9]. However, one of the drawbacks of this flap is related to donor site morbidity, since the donor site defect which remains on the dorsum of the index finger, requires skin graft for coverage from a second donor site [10]. This additional skin graft harvest might be associated with further complications [10].

\footnotetext{
* Corresponding author: Dimitrios Giotis

Orthopaedic Department, General Hospital of Ioannina “G. Hatzikosta”, Ioannina, Greece.
}

Copyright (C) 2021 Author(s) retain the copyright of this article. This article is published under the terms of the Creative Commons Attribution Liscense 4.0. 
The aim of the current study is to report a case of a thumb soft tissue defect which was treated with a modified dorsal metacarpal artery (DMA) flap without any need for extra skin graft from another donor site. In this surgical technique, a single modified incision was performed along the ulnar border of the second metacarpal, not only to harvest the first DMA flap to cover the thumb defect, but also to raise a second DMA flap which was used for the coverage of the defect in the index finger. A direct closure of the donor site defect on the dorsum of the metacarpal was achieved owing to the laxity of the dorsal skin [11].

\section{Material and methods}

A 65-year-old man presented to the Emergency Room (ER) with a crush injury to the thumb. The patient was conscious and haemodynamically normal, without any further injuries. The clinical examination revealed a $34 \mathrm{x} 16 \mathrm{~mm}$ skin defect on the dorsal side of the thumb over its proximal and distal phalanx, extending circumferentially to the ipsilateral thenar, with exposure of subjacent bone and tendon (Figure 1). The extensor pollicis longus (EPL) tendon was found intact and the patient was fully capable to both flex and extend the interphalangeal (IP) joint. In parallel, no other severe neurovascular damage was observed as the thumb was warm and adequately perfused with intact sensation on the tip of the thumb. Radiographically, there was no sign of fracture.

Primary management in the ER started with wound cleansing and dressing with moist saline gauze. Preoperatively, broad-spectrum antibiotics along with tetanus immunoglobulin were administered. The surgical intervention was performed under general anesthesia, within six hours. Initially, irrigation and debridement of the wound was performed followed by a meticulous intraoperative evaluation which indicated that EPL tendon and capsules of the IP and metacarpophalangeal (MP) joints were uninjured.

In order to cover the skin defect, the first DMA flap was marked on the dorsum of the proximal phalanx of the index finger to match the size and shape of the defect on the thumb. Afterwards, a modified c-shaped incision was used along the ulnar border of the second metacarpal with the convex side of the curve looking radially, in contrast to the traditional incision along the radial border of the second metacarpal. Dissection of the first DMA flap started from distal to proximal and from ulnar to radial which included the periosteum of the second metacarpal bone and the fascia of the ulnar head of the first dorsal interosseous muscle. In this way, the DMA flap was raised along with its pedicle, which included the first DMA, its concomitant veins and a branch of the superficial radial nerve, and subsequently transferred to cover the thumb defect through a subcutaneous tunnel. Then, a second flap was raised from the same incision pedicled on a distal cutaneous branch of the second DMA. This second DMA perforator flap was rotated and advanced to cover the defect on the dorsum of the index finger (Figure 2). Finally, linear donor site closure was achieved with nylon sutures and the wrist was immobilized in extension for 2 weeks (Figure 3a/b). Sutures were removed 3 weeks postoperatively (Figure $4 a / b)$.

\section{Results and discussion}

At 3 months post-injury, the flaps had survived without complications and the patient had regained active range of motion of the fingers, being satisfied with the cosmetic outcome. Patient's digit function and sensation over the flap were assessed using the Michigan Hand Outcomes Questionnaire (MHQ) [12] and the 2-point discrimination (2-PD) test, respectively. Specifically, the patient had a mean score of 86 ( $0=$ worst result, $100=$ best result) on the MHQ. Regarding the 2-PD test, the 2-PD in the injured thumb was $3.5 \mathrm{~mm}$ as compared to $3.1 \mathrm{~mm}$ in the uninjured (Figure $5 \mathrm{a} / \mathrm{b})$.

Reconstruction of extensive soft tissue defects of the thumb with exposure of bone, tendons or joints, is considered challenging for surgeons $[2,13]$. Several surgical options have been reported in the literature including local, regional and free flaps $[3,13]$. Regional flaps harvested from the forearm offer satisfying coverage but they lead to donor site morbidity $[14,15]$. Free flaps require microsurgical experience, long operative time and the final sensory outcome is not always as expected $[1,16]$. On the other hand, local flaps offer advanced esthetic result as the defect is replaced by "similar" tissue. Especially the DMA flaps outmatch as they offer extra amount of soft tissue availability [3, 6, 9, 17-18].

Mostly, in DMA flaps, both the first and second DMA flaps are harvested from the dorsum of the hand along with their artery, veins and a branch of the radial nerve that maintains sensation [5]. The first and second DMAs are frequently constant vessels but their anatomy may vary [19]. However, cases where these vessels were absent have also been recorded [20]. This might complicate the operation, as the skeletonization and dissection of the DMA may be extremely difficult for a surgeon. In such cases where the first DMA is missing, an alternative procedure can be performed by 
raising a second DMA flap from the middle finger to cover the thumb defect [21]. In order to avoid contiguous problems, the use of preoperative Doppler ultrasound to verify the existence of the DMAs, is advocated [11].

Regarding the anatomy, the first DMA (FDMA) usually consists of three branches the radial DMA (DMAr), the intermediate (DMAi) and the ulnar (DMAu) [22]. The DMAu is the main vessel that provides blood supply to the first DMA (FDMA) flap and when it is absent the flap is nourished by dorsopalmar anastomoses [3, 11]. The second DMA consists of cutaneous branches that seem to be clustered at three positions [23]. In our case, the DMAu was present intraoperatively and the FDMA flap was raised pedicled on it, whereas a distal cutaneous branch perfused the second DMA flap and the rest cutaneous branches were ligated.

With the use of modified first DMA flaps, even greater defects regarding the entire dorsal or palmar surface of the thumb can be adequately covered [4,13]. In the traditional technique modified by Foucher [24], the defect on the dorsum of the index requires a skin graft for coverage which may result in both donor site morbidity and restriction of movement in the metacarpophalangeal (MP) joint due to the bulk of the skin graft and scarring $[1,14,25]$.

In our case, in order to avoid the associated drawbacks that accompany a skin graft transfer, a modified incision was performed for the harvest of the second DMA flap to cover the defect on the dorsum of the index finger. Apart from the advantages in terms of soft tissue availability, operative time and single stage reconstruction, the cosmetic outcome due to linear skin closure, was satisfactory [14]. Nonetheless, complications have been reported in the literature in similar cases such as partial or complete flap loss attributed to arterial insufficiency due to improper flap elevation technique or small caliber DMAs $[11,14,26]$. Furthermore, venous congestion is another complication mainly appearing in reverse DMA flaps where there is concomitant reverse blood flow [27]. Lastly, loss of sensation that is typically caused by injury to the branches of the radial nerve during flap dissection, is rarely indicated $[4,11]$.

\section{Legends}

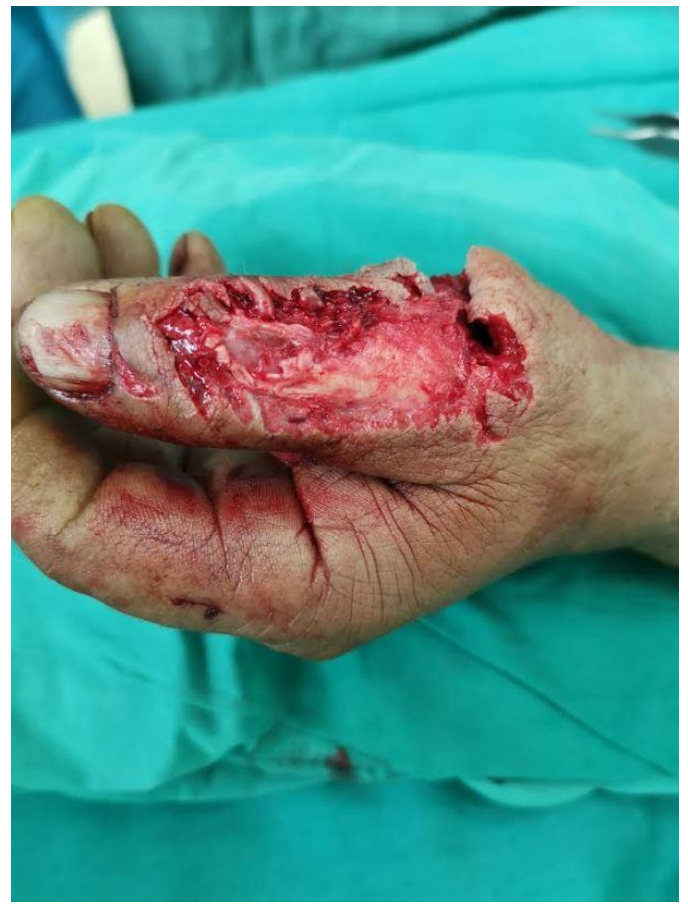

Figure 1 Skin defect on the dorsal side of the thumb

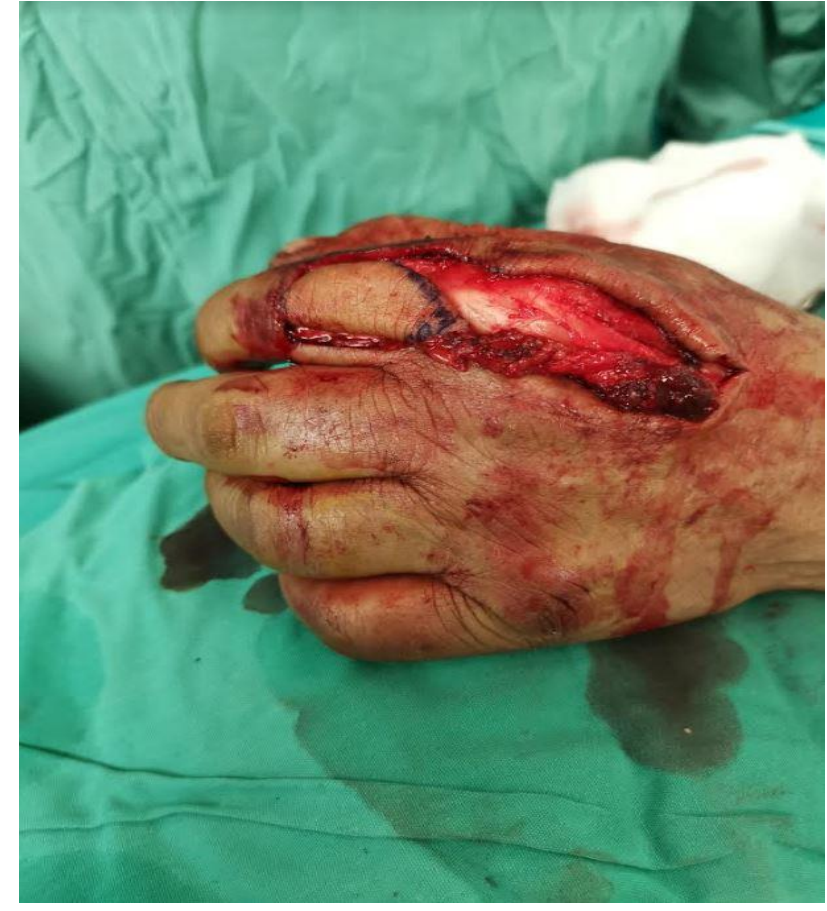

Figure 2 DMA flap harvested and transferred to cover the thumb defect 
International Journal of Science and Research Archive, 2021, 03(02), 107-112
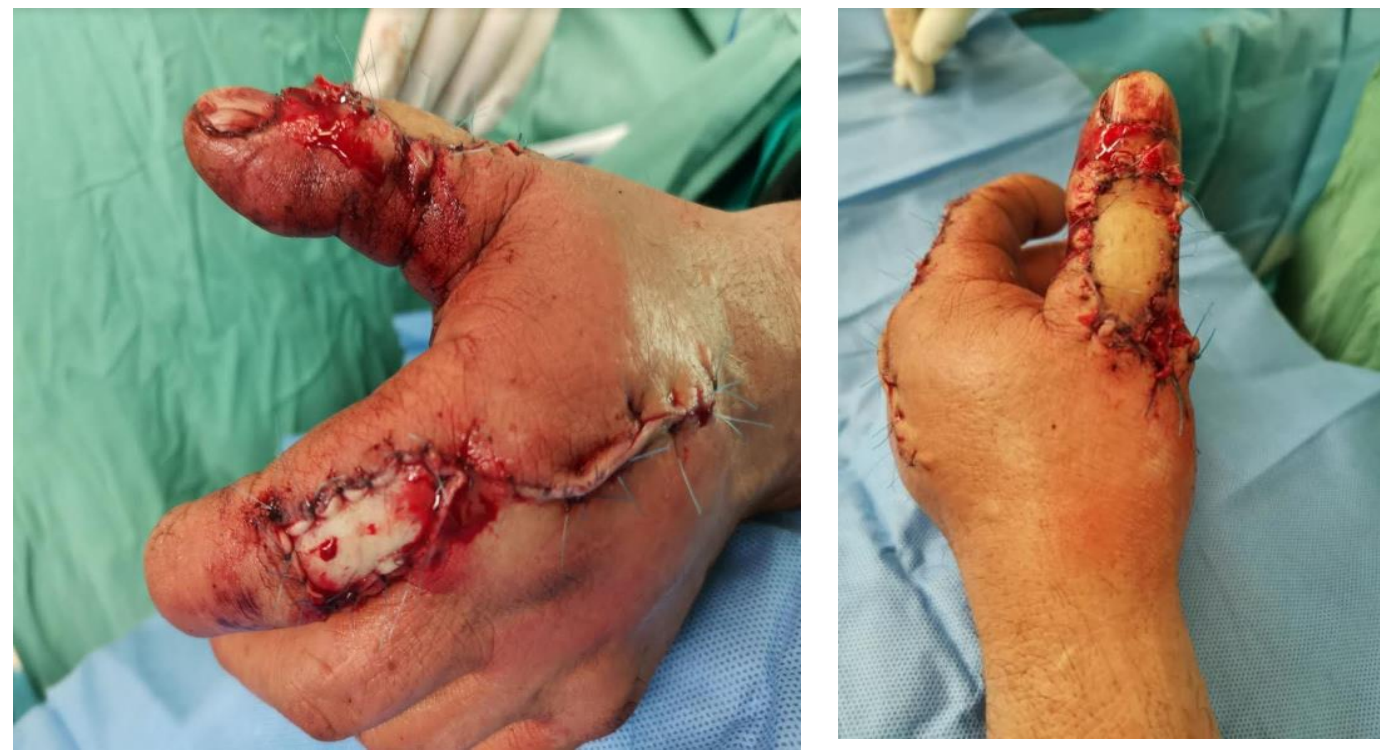

Figure 3a/b Donor site closure
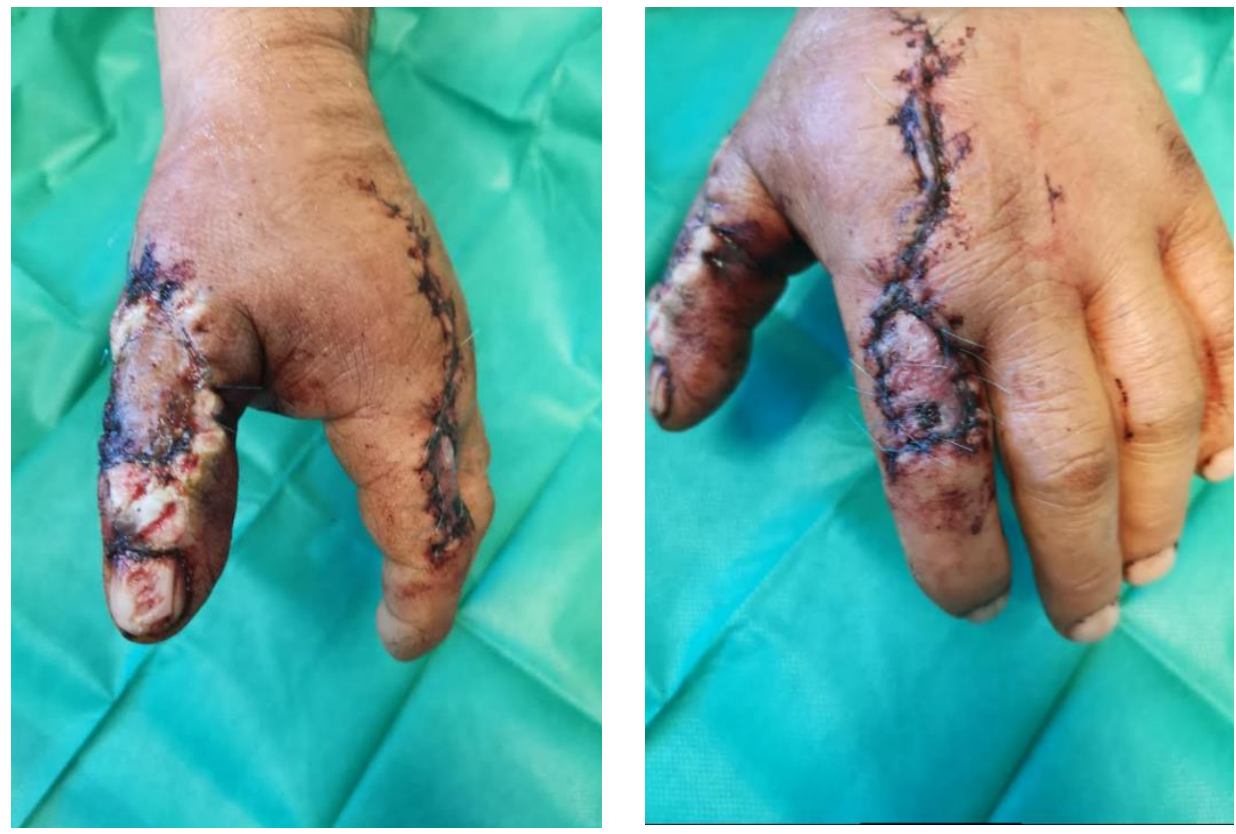

Figure $4 \mathbf{a} / \mathbf{b}$ Removal of sutures at 3 weeks post-op. 

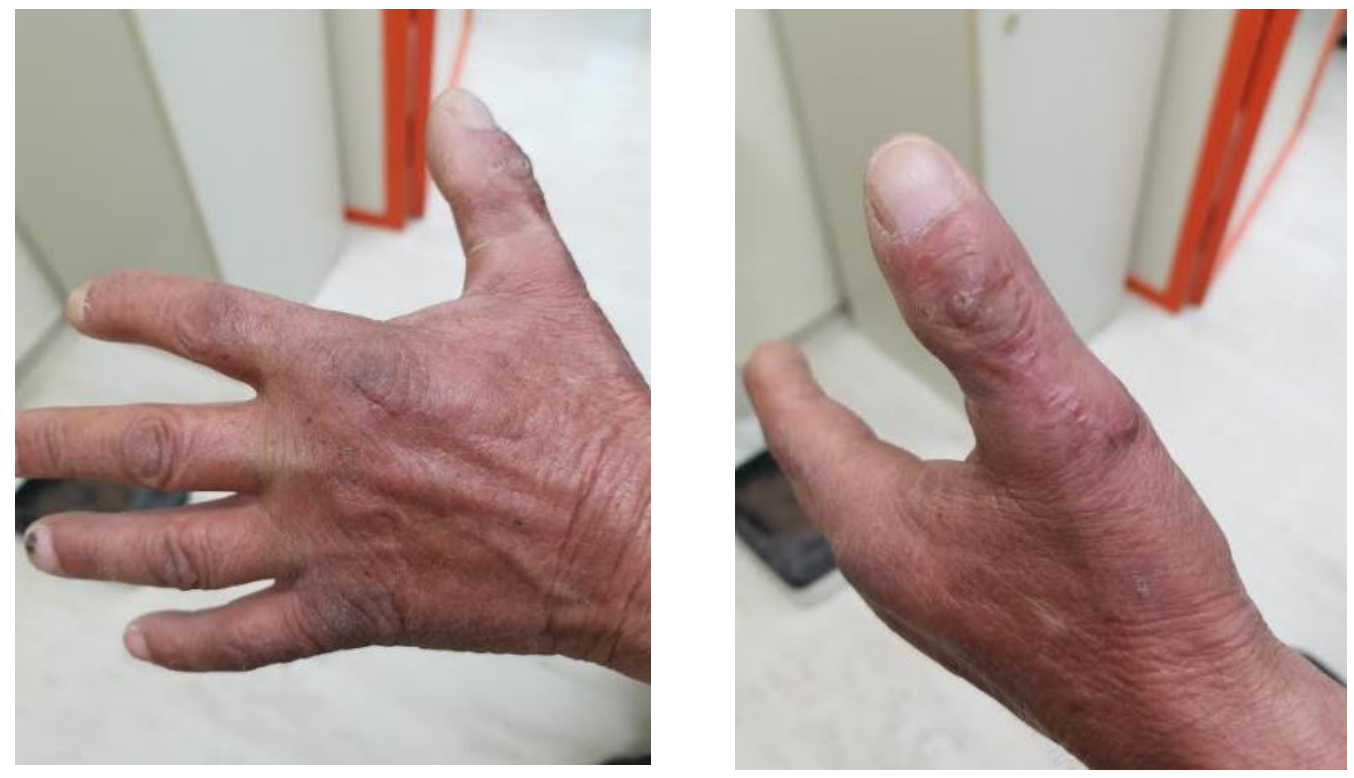

Figure 5a/b Final clinical and cosmetic outcome.

\section{Conclusion}

This modified incision allows simultaneous elevation of two DMA flaps for closure of both the soft tissue defect of the primary injury as well as the donor site defect on the dorsum of the index. Overall, this technique prevents the associated complications that may accompany the application of remote skin grafts and has an acceptable functional and cosmetic outcome.

\section{Compliance with ethical standards}

\section{Disclosure of conflict of interest}

All authors declare that they have no competing interests.

\section{Statement of informed consent}

The patient gave his informed consent for participation in accordance with our Institutional Review Board procedures.

\section{References}

[1] Ghoraba SM, Mahmoud WH. Outcome of thumb reconstruction using the first dorsal metacarpal artery island flap. World J Plast Surg. 2018; 7(2):151-8.

[2] Pet MA, Ko JH, Vedder NB. Reconstruction of the traumatized thumb. Plast Reconstr Surg. 2014; 134(6):12351245.

[3] Alexander L. Dorsal metacarpal artery flap: an underrated workhorse flap for reconstruction of dorsal finger defects. Cureus. 2020; 12(10):e11251.

[4] Webster N, Saint-Cyr M. Flaps based on the dorsal metacarpal artery. Hand Clin. 2020; 36(1):75-83.

[5] Muyldermans T, Hierner R. First dorsal metacarpal artery flap for thumb reconstruction: A retrospective clinical study. Strategies Trauma Limb Reconstr. 2009; 4:27-33.

[6] Couceiro J, de Prado M, Menendez G, Manteiga Z. The first dorsal metacarpal artery flap family: A review. Surg J (N Y). 2018; 4(4):e215-e219.

[7] Zhang X, Shao X, Ren C, Zhang Z, Wen S, Sun J. Reconstruction of thumb pulp defects using a modified kite flap. J Hand Surg Am. 2011; 36(10):1597-1603. 
[8] Zhang X, Shao X, Shen Q, Yu Y, Li Y, Fan A. Use of the first dorsal metacarpal artery-based fascial flap for reconstruction of small defects on the dorsum of the hands. J Hand Surg Am. 2019; 44(12):1096.e1-1096.e6.

[9] Ege A, Tuncay I, Ercetin O. Foucher's first dorsal metacarpal artery flap for thumb reconstruction: Evaluation of 21 cases. Isr Med Assoc J. 2002; 4(6):421-3.

[10] Jaquet Y, Enepekides DJ, Torgerson C, Higgins KM. Radial forearm free flap donor site morbidity: Ulnar-based transposition flap vs split-thickness skin graft. Arch Otolaryngol Head Neck Surg. 2012; 138:38-43.

[11] Sebastin SJ, Mendoza RT, Chong AKS, Peng YP, Ono S, Chung KC, Lim AYT. Application of the dorsal metacarpal artery perforator flap for resurfacing soft-tissue defects proximal to the fingertip. Plast Reconstr Surg. 2011; 128(3):166e-178e.

[12] Chung KC, Pillsbury MS, Walters MR, Hayward RA. Reliability and validity testing of the Michigan Hand Outcomes Questionnaire. J Hand Surg Am. 1998; 23:575-87.

[13] Doğan F, Çoruh A. Novel biogeometric designs of first dorsal metacarpal artery flap in hand reconstruction. J Burn Care Res. 2014; 35(6):e399-e405.

[14] Chi Z, Lin D, Chen Y, Xue J, Li S, Chu T, Li Z. Routine closure of the donor site with a second dorsal metacarpal artery flap to avoid the use of a skin graft after harvest of a first dorsal metacarpal artery flap. J Plast Reconstr Aesthet Surg. 2018; 71(6):870-5.

[15] Demiri EC, Dionyssiou DD, Pavlidis LC, Papas AV, Kostogloudis NH, Lykoudis EG. Soft tissue reconstruction of the thumb with the dorsoradial forearm flap. J Hand Surg Eur Vol. 2013; 38(4):412-7.

[16] Adani R, Cardon LJ, Castagnetti C, Pinelli M. Distal thumb reconstruction using a mini wrap-around flap from the great toe. J Hand Surg Br. 1999; 24(4):437-42.

[17] Luan Z, Liu B, Jiang H, Gao F, Yang B. Reverse first dorsal metacarpal artery flap repair of a right thumb epidermis granuloma: A case study. Oncol Lett. 2019; 18(3):2384-87.

[18] Spyropoulou GA, Shih HS, Jeng SF. Free pulp transfer for fingertip reconstruction-the algorithm for complicated Allen fingertip defect. Plast Reconstr Surg Glob Open. 2016; 3(12):e584.

[19] Dauphin N, Casoli V. The dorsal metacarpal arteries: Anatomical study. Feasibility of pedicled metacarpal bone flaps. J Hand Surg Eur Vol. 2011; 36(9):787-94.

[20] Gupta C, Ray B, Dsouza AS, Nair N, Pai SR, Manju M. A morphological study of variations in the branching pattern and termination of the radial artery. Singapore Med J. 2012; 53(3):208-11.

[21] Zhang X, He Y, Shao X, Li Y, Wen S, Zhu H. Second dorsal metacarpal artery flap from the dorsum of the middle finger for coverage of volar thumb defect. J Hand Surg Am. 2009; 34(8):1467-73.

[22] Sherif MM. First dorsal metacarpal artery flap in hand reconstruction. I. Anatomical study. J Hand Surg Am. 1994; 19(1):26-31.

[23] Liu P, Deng Z, Zhang T, Li X. Anatomical characteristics of cutaneous branches extending from the second dorsal metacarpal artery. Front Bioeng Biotechnol. 2020; 8:995.

[24] Foucher G, Braun JB. A new island flap transfer from the dorsum of the index to the thumb. Plast Reconstr Surg. $1979 ; 63(3): 344-9$.

[25] Koch H, Bruckmann L, Hubmer M, Scharnagl E. Extended reverse dorsal metacarpal artery flap: clinical experience and donor site morbidity. J Plast Reconstr Aesthet Surg. 2007; 60(4):349-55.

[26] Balan JR, Mathew S, Kumar P, Vardhan H, Francis A, Aniljith VG, Gopal R. The reverse dorsal metacarpal artery flap in finger reconstruction: A reliable choice. Indian J Plast Surg. 2018; 51(1):54-9.

[27] Shen H, Shen Z, Wang Y, Zhang K, Zhang Z, Dai X. Extended reverse dorsal metacarpal artery flap for coverage of finger defects distal to the proximal interphalangeal joint. Ann Plast Surg. 2014; 72(5):529-36. 